IJMMS 31:4 (2002) 201-213

PII. S0161171202005860

http://ijmms.hindawi.com

(c) Hindawi Publishing Corp.

\title{
ON THE SOLVABILITY OF PARABOLIC AND HYPERBOLIC PROBLEMS WITH A BOUNDARY INTEGRAL CONDITION
}

\author{
ABDELFATAH BOUZIANI \\ Received 22 September 2000
}

\begin{abstract}
We prove the existence, uniqueness, and the continuous dependence of a generalized solution upon the data of certain parabolic and hyperbolic equations with a boundary integral condition. The proof uses a functional analysis method based on a priori estimates established in nonclassical function spaces and on the density of the range of the linear operator associated to the abstract formulation of the studied problem.
\end{abstract}

2000 Mathematics Subject Classification: 35K20, 35L20, 35B45, 46E30, 35D05, 35B30.

1. Introduction. The aim of this paper is to develop the a priori estimates method for the mixed problems, which involve integral(s) over the spatial domain of a function of the desired solution. The presence of integral terms in boundary conditions can, in general, greatly complicate the application of standard functional or numerical techniques. To avoid this difficulty, we introduce a nonclassical function space, in which we take the scalar product of the considered equation and the same operator of multiplication used to establish the a priori estimate for classical mixed problems related to the same equation. We note that the construction of such operators is the crucial step to establish the a priori estimates. We apply this idea to a mixed problem for second-order parabolic equation which combines Neumann and integral conditions:

$$
\begin{gathered}
\mathscr{L} v=\frac{\partial v}{\partial t}-\frac{\partial}{\partial x}\left(a(x, t) \frac{\partial v}{\partial x}\right)+b(x, t) v=\mathrm{f}(x, t), \quad \alpha<x<\beta, 0<t<T, \\
\ell v=v(x, 0)=\Phi(x), \quad \alpha<x<\beta, \\
\frac{\partial v(\alpha, t)}{\partial x}=\mu(t), \quad \int_{\alpha}^{\beta} v(x, t) d x=m(t), \quad 0<t<T,
\end{gathered}
$$

where $\Phi, \mu, m, a, b, f$ are known functions, and $\alpha, \beta, T$ are given constants.

This problem is choused because it has been studied, in special form, quite extensively, both numerically and analytically, but using complicate ways. Cannon and van der Hoek [6] considered the coupling of the numerical solution of a Volterra integral equation of the second kind to a finite difference scheme for the heat equation. Cannon, Esteva, and van der Hoek [5] considered a semi-discrete Galerkin method based on rather complicated weak form of the mixed problem which requires the solution of a system of linear Volterra integrodifferential equations of the second kind. They also analyzed a fully discrete Crank-Nicolson Galerkin method. Fairweather and Saylor [7] converted a similar problem of (1.1), (1.2), and (1.3) to one with separated boundary conditions and, in a more straightforward manner than the Crank-Nicolson 
Galerkin approach in [5], they considered a finite difference technique based on Keller's box scheme [8]; the price one pays for this proof is an increase of one in the number of partial differential equations for each integral condition. Lardner [9] has devised a way to cast the characteristic polynomial of resulting matrix to a trigonometric polynomial in seeking its eigenvalues. He proposed first that eigenvalues bear the form $\lambda=2 \cos \theta-2$. Then it was detected by numerical computations that for matrices of order less than or equal to 20 all eigenvalues are indeed of the proposed form. Although the author did not provide a rigorous proof of his claims on the desired locations of eigenvalues. Shi [10] uses a variational formulation in which the integral condition does not explicitly appear to prove the well-posedness. In his proof, he established an a priori estimate based on an interpolation inequality for norms of weighted fractional Sobolev spaces, and on the Fourier transform.

Problem (1.1), (1.2), and (1.3) arises in many physical processes. For example, the reader is referred to $[4,5,7]$.

In order to show how the method can be developed with other classes of problems together with integral condition(s), we study, in parallel, a mixed problem for a second-order hyperbolic equation which combines Neumann condition with an integral condition, that is,

$$
\begin{gathered}
\mathscr{L}^{\prime} v=\frac{\partial^{2} v}{\partial t^{2}}-\frac{\partial}{\partial x}\left(a(x, t) \frac{\partial v}{\partial x}\right)+b(x, t) v=\mathrm{f}(x, t), \quad \alpha<x<\beta, 0<t<T, \\
\ell_{1} v=v(x, 0)=\Phi(x), \quad \ell_{2} v=\frac{\partial v(x, 0)}{\partial t}=\Psi(x), \quad \alpha<x<\beta, \\
\frac{\partial v(\alpha, t)}{\partial x}=\mu(t), \quad \int_{\alpha}^{\beta} v(x, t) d x=m(t), \quad 0<t<T .
\end{gathered}
$$

The paper is organized as follows. We begin by stating the precise assumptions of the functions involved in the posed problems, by introducing certain function spaces which are often used in the next sections, by defining a generalized solution, and by giving the main results of the paper. In Section 3, we first establish a priori estimates, then the uniqueness and continuous dependence are direct consequences. The existence of the solution is proved in Section 4.

2. Preliminaries and main results. We begin with the following assumptions.

Assumption 2.1. There are positive constants $c_{i}, i=0,1,2,3,4$, such that, for $(x, t) \in[\alpha, \beta] \times[0, T]$,

$$
0<c_{0} \leq a(x, t) \leq c_{1}, \quad\left|\frac{\partial a}{\partial t}\right| \leq c_{2}, \quad\left|\frac{\partial a}{\partial x}\right| \leq c_{3}, \quad|b(x, t)| \leq c_{4} .
$$

AsSUMPTION 2.2. We assume the following compatibility conditions:

$$
\begin{gathered}
\frac{d \Phi(\alpha)}{d x}=\mu(0), \quad \int_{\alpha}^{\beta} \Phi(x) d x=m(0) \\
{\left[\frac{d \Phi(\alpha)}{d x}=\mu(0), \frac{d \Psi(\alpha)}{d x}=\mu^{\prime}(0), \int_{\alpha}^{\beta} \Phi(x) d x=m(0), \int_{\alpha}^{\beta} \Psi(x) d x=m^{\prime}(0)\right] .}
\end{gathered}
$$


Since the boundary conditions are inhomogeneous, we construct a function

$$
U(x, t)=(x-\alpha)\left(1-\frac{3(x-\alpha)}{2(\beta-\alpha)}\right) \mu(t)+\frac{3(x-\alpha)^{2}}{(\beta-\alpha)^{3}} m(t)
$$

and introduce a new function $u(x, t)=v(x, t)-U(x, t)$. Then problem (1.1), (1.2), and (1.3), [(1.4), (1.5), and (1.6)], can be formulated as

$$
\begin{gathered}
\mathscr{L} u=\frac{\partial u}{\partial t}-\frac{\partial}{\partial x}\left(a(x, t) \frac{\partial u}{\partial x}\right)+b(x, t) u=\mathrm{f}(x, t)-\mathscr{L} U=f(x, t), \\
\ell u=u(x, 0)=\Phi(x)-\ell U=\varphi(x), \\
\frac{\partial u(\alpha, t)}{\partial x}=0, \quad \int_{\alpha}^{\beta} u(x, t) d x=0, \\
{\left[\mathscr{L}^{\prime} u=\frac{\partial^{2} u}{\partial t^{2}}-\frac{\partial}{\partial x}\left(a(x, t) \frac{\partial u}{\partial x}\right)+b(x, t) u=\mathrm{f}(x, t)-\mathscr{L}^{\prime} U=f(x, t),\right.} \\
\ell_{1} u=u(x, 0)=\Phi(x)-\ell_{1} U=\varphi(x), \\
\ell_{2} u=\frac{\partial u(x, 0)}{\partial t}=\Psi(x)-\ell_{2} U=x(x), \\
\left.\frac{\partial u(\alpha, t)}{\partial x}=0, \quad \int_{\alpha}^{\beta} u(x, t) d x=0\right] .
\end{gathered}
$$

Assumption 2.3. We assume the compatibility conditions

$$
\begin{gathered}
\frac{d \varphi(\alpha)}{d x}=\mu(0), \quad \int_{\alpha}^{\beta} \varphi(x) d x=0 \\
{\left[\frac{d \varphi(\alpha)}{d x}=0, \frac{d \varkappa(\alpha)}{d x}=0, \int_{\alpha}^{\beta} \varphi(x) d x=0, \int_{\alpha}^{\beta} \varkappa(x) d x=0\right]}
\end{gathered}
$$

We introduce the function spaces, which we need in our investigation. Let $L^{2}(\alpha, \beta)$ and $L^{2}\left(0, T ; L^{2}(\alpha, \beta)\right)$ be the standard function spaces. We denote by $C_{0}(\alpha, \beta)$ the vector space of continuous functions with compact support in $(\alpha, \beta)$. Since such functions are Lebesgue integrable with respect to $d x$, we can define on $C_{0}(\alpha, \beta)$ the bilinear form given by

$$
((u, w))=\int_{\alpha}^{\beta} \mathfrak{J}_{x} u \cdot \mathfrak{I}_{x} w d x
$$

where $\mathfrak{I}_{x} u=\int_{\alpha}^{x} u(\xi, \cdot) d \xi$. The bilinear form (2.11) is considered as a scalar product on $C_{0}(\alpha, \beta)$ for which $C_{0}(\alpha, \beta)$ is not complete. 
DEFINITION 2.4. We denote by $B_{2}^{1}(\alpha, \beta)$ a completion of $C_{0}(\alpha, \beta)$ for the scalar product (2.11), which is denoted $(\cdot, \cdot)_{B_{2}^{1}(\alpha, \beta)}$, called the Bouziani space or the space of square integrable primitive functions on $(\alpha, \beta)$. By the norm of function $u$ from $B_{2}^{1}(\alpha, \beta)$, we understand the nonnegative number:

$$
\|u\|_{B_{2}^{1}(\alpha, \beta)}=\sqrt{(u, u)_{B_{2}^{1}(\alpha, \beta)}}=\left\|\mathfrak{I}_{x} u\right\|_{L^{2}(\alpha, \beta)} .
$$

For $u \in L^{2}(\alpha, \beta)$, we have the elementary inequality

$$
\|u\|_{B_{2}^{1}(\alpha, \beta)} \leq \frac{|\alpha-\beta|}{\sqrt{2}}\|u\|_{L^{2}(\alpha, \beta)}
$$

We denote by $L^{2}\left(0, T ; B_{2}^{1}(\alpha, \beta)\right)$ the space of functions which are square integrable in the Bochner sense, with the scalar product

$$
(u, w)_{L^{2}\left(0, T ; B_{2}^{1}(\alpha, \beta)\right)}=\int_{0}^{T}(u(\cdot, t), w(\cdot, t))_{B_{2}^{1}(\alpha, \beta)} d t .
$$

Since the space $B_{2}^{1}(\alpha, \beta)$ is a Hilbert space, it can be shown that $L^{2}\left(0, T ; B_{2}^{1}(\alpha, \beta)\right)$ is a Hilbert space as well. The set of all continuous abstract functions in $[0, T]$ equipped with the norm

$$
\sup _{0 \leq T \leq T}\|u(\cdot, \tau)\|_{B_{2}^{1}(\alpha, \beta)}
$$

is denoted $C\left(0, T ; B_{2}^{1}(\alpha, \beta)\right)$.

The problem (2.4), (2.5), and (2.6) [(2.7), (2.8), and (2.9)] is equivalent to the operator equation $L u=(f, \varphi)\left[L^{\prime} u=(f, \varphi, \varkappa)\right]$, where $L=(\mathscr{L}, \ell)\left[L^{\prime}=\left(\mathscr{L}^{\prime}, \ell_{1}, \ell_{2}\right)\right]$ acts from $B\left[B^{\prime}\right]$ to $F\left[F^{\prime}\right]$, where $B$ is the space of functions $u$ belonging to $L^{2}\left(0, T ; L^{2}(\alpha, \beta)\right) \cap$ $C\left(0, T ; B_{2}^{1}(\alpha, \beta)\right)$ and satisfying conditions (2.6), $B^{\prime}$ is the Banach space of functions $u \in L^{2}\left(0, T ; B_{2}^{1}(\alpha, \beta)\right)$ having the finite norm

$$
\|u\|_{B^{\prime}}=\left(\|u\|_{C\left(0, T ; L^{2}(\alpha, \beta)\right)}^{2}+\left\|\frac{\partial u}{\partial t}\right\|_{C\left(0, T ; B_{2}^{1}(\alpha, \beta)\right)}^{2}\right)^{1 / 2}
$$

and verifying conditions (2.9), $F=L^{2}\left(0, T ; B_{2}^{1}(\alpha, \beta)\right) \times B_{2}^{1}(\alpha, \beta)$ and $F^{\prime}=L^{2}(0, T$; $\left.B_{2}^{1}(\alpha, \beta)\right) \times L^{2}(\alpha, \beta) \times B_{2}^{1}(\alpha, \beta)$. The domain $D(L)$ of $L$ is the set of all $u \in$ $L^{2}\left(0, T ; B_{2}^{1}(\alpha, \beta)\right)$ for which $\partial u / \partial t, \partial u / \partial x, \partial^{2} u / \partial x^{2} \in L^{2}\left(0, T ; B_{2}^{1}(\alpha, \beta)\right)$ and satisfying (2.6), and the domain $D\left(L^{\prime}\right)$ of $L^{\prime}$ is the set of all $u \in L^{2}\left(0, T ; B_{2}^{1}(\alpha, \beta)\right)$ for which $\partial u / \partial t, \partial^{2} u / \partial t^{2}, \partial u / \partial x, \partial^{2} u / \partial x^{2} \in L^{2}\left(0, T ; B_{2}^{1}(\alpha, \beta)\right)$ and fulfilling (2.9). Let $\bar{L}\left[\overline{L^{\prime}}\right]$ be the closure of operator $L\left[L^{\prime}\right]$.

DEFINITION 2.5. A solution of the operator equation

$$
\bar{L} u=(f, \varphi)\left[\overline{L^{\prime}} u=(f, \varphi, \varkappa)\right]
$$

is called a generalized solution of the problem (2.4), (2.5), and (2.6) [(2.7), (2.8), and (2.9)]. 
The main purpose of the present paper is to prove the following theorem.

TheOREм 2.6. Assume that Assumptions 2.1 and 2.3 are satisfied. Then the solution of problem (2.4), (2.5), (2.6) [(2.7), (2.8), and (2.9)] satisfies the following a priori estimate:

$$
\begin{gathered}
\|u\|_{B} \leq c\|L u\|_{F}, \quad \forall u \in D(L), \\
{\left[\|u\|_{B^{\prime}} \leq c^{\prime}\left\|L^{\prime} u\right\|_{F^{\prime}}, \quad \forall u \in D\left(L^{\prime}\right)\right],}
\end{gathered}
$$

where $c$ and $c^{\prime}$ are positive constants independent of $u$.

THEOREM 2.7. Under the assumptions of Theorem 2.6, problem (2.4), (2.5), and (2.6) [(2.7), (2.8), and (2.9)] admits a unique generalized solution in the sense of Definition 2.5, satisfying the following properties:

$$
\begin{gathered}
u \in L^{2}\left(0, T ; L^{2}(\alpha, \beta)\right) \cap C\left(0, T ; B_{2}^{1}(\alpha, \beta)\right), \\
{\left[u \in C\left(0, T ; L^{2}(\alpha, \beta)\right), \frac{\partial u}{\partial t} \in C\left(0, T ; B_{2}^{1}(\alpha, \beta)\right)\right] .}
\end{gathered}
$$

The proof of the above theorems will be given in the next sections.

3. Uniqueness and continuous dependence. In this section, we prove Theorem 2.6 in which we establish an a priori estimate for a solution of problem (2.4), (2.5), and (2.6) [(2.7), (2.8), and (2.9)]. Then, the uniqueness and continuous dependence of the generalized solution upon the data are direct consequences.

Proof OF TheOrem 2.6. Taking the scalar product in $B_{2}^{1}(\alpha, \beta)$ of (2.4) and [(2.7)] and $u[\partial u / \partial t]$, we have

$$
\begin{gathered}
\left(\frac{\partial u(\cdot, t)}{\partial t}, u(\cdot, t)\right)_{B_{2}^{1}(\alpha, \beta)}-\left(\frac{\partial}{\partial x}\left(a \frac{\partial u(\cdot, t)}{\partial x}\right), u(\cdot, t)\right)_{B_{2}^{1}(\alpha, \beta)} \\
+(b u(\cdot, t), u(\cdot, t))_{B_{2}^{1}(\alpha, \beta)}=(f(\cdot, t), u(\cdot, t))_{B_{2}^{1}(\alpha, \beta)}, \\
{\left[\left(\frac{\partial^{2} u(\cdot, t)}{\partial t^{2}}, \frac{\partial u(\cdot, t)}{\partial t}\right)_{B_{2}^{1}(\alpha, \beta)}-\left(\frac{\partial}{\partial x}\left(a \frac{\partial u(\cdot, t)}{\partial x}\right), \frac{\partial u(\cdot, t)}{\partial t}\right)_{B_{2}^{1}(\alpha, \beta)}\right.} \\
\left.+\left(b u(\cdot, t), \frac{\partial u(\cdot, t)}{\partial t}\right)_{B_{2}^{1}(\alpha, \beta)}=\left(f(\cdot, t), \frac{\partial u(\cdot, t)}{\partial t}\right)_{B_{2}^{1}(\alpha, \beta)}\right] .
\end{gathered}
$$

In light of conditions (2.6) [(2.9)], integrating by parts the left-hand side of (3.1) [(3.2)], we obtain

$$
\begin{aligned}
& \int_{\alpha}^{\beta} a(x, t) u^{2}(x, t) d x+\frac{1}{2} \frac{\partial}{\partial t}\|u(\cdot, t)\|_{B_{2}^{1}(\alpha, \beta)}^{2} \\
&=(f(\cdot, t), u(\cdot, t))_{B_{2}^{1}(\alpha, \beta)}+\int_{\alpha}^{\beta}\left(\frac{\partial a}{\partial x} u+\mathfrak{J}_{x}(b u)\right) \mathfrak{J}_{x} u d x,
\end{aligned}
$$




$$
\begin{aligned}
& {\left[\frac{1}{2} \frac{\partial}{\partial t}\left\{\int_{\alpha}^{\beta} a(x, t) u^{2}(x, t) d x+\left\|\frac{\partial u(\cdot, t)}{\partial t}\right\|_{B_{2}^{1}(\alpha, \beta)}^{2}\right\}\right.} \\
& \left.\quad=\left(f(\cdot, t), \frac{\partial u(\cdot, t)}{\partial t}\right)_{B_{2}^{1}(\alpha, \beta)}+\int_{\alpha}^{\beta}\left(\frac{\partial a}{\partial x} u+\mathfrak{J}_{x}(b u)\right) \mathfrak{J}_{x} \frac{\partial u}{\partial t} d x+\frac{1}{2} \int_{\alpha}^{\beta} \frac{\partial a}{\partial t} u^{2} d x\right] .
\end{aligned}
$$

Estimating the right-hand side of the equality (3.3) [(3.4)], we get

$$
\begin{aligned}
& 2 \int_{\alpha}^{\beta} a(x, t) u^{2}(x, t) d x+\frac{\partial}{\partial t}\|u(\cdot, t)\|_{B_{2}^{1}(\alpha, \beta)}^{2} \\
& \leq\|f(\cdot, t)\|_{B_{2}^{1}(\alpha, \beta)}^{2}+\left(1+\frac{1}{\varepsilon_{2}}\right)\|u(\cdot, t)\|_{B_{2}^{1}(\alpha, \beta)}^{2} \\
&+\frac{1}{\varepsilon_{1}}\|u(\cdot, t)\|_{L^{2}(\alpha, \beta)}^{2}+\varepsilon_{1} \int_{\alpha}^{\beta}\left(\frac{\partial a}{\partial x}\right)^{2}\left(\mathfrak{J}_{x} u\right)^{2} d x \\
&+\varepsilon_{2} \int_{\alpha}^{\beta}\left(\mathfrak{J}_{x}(b u)\right)^{2} d x, \\
& {\left[\frac{\partial}{\partial t}\left\{\int_{\alpha}^{\beta} a(x, t) u^{2}(x, t) d x+\left\|\frac{\partial u(\cdot, t)}{\partial t}\right\|_{B_{2}^{1}(\alpha, \beta)}^{2}\right\}\right.} \\
& \leq\|f(\cdot, t)\|_{B_{2}^{1}(\alpha, \beta)}^{2}+\left(1+\frac{1}{\varepsilon_{3}}+\frac{1}{\varepsilon_{4}}\right) \|^{\frac{\partial u(\cdot, t)}{\partial t} \|_{B_{2}^{1}(\alpha, \beta)}^{2}} \\
&\left.\quad+\int_{\alpha}^{\beta} \frac{\partial a}{\partial t} u^{2} d x+\varepsilon_{3} \int_{\alpha}^{\beta}\left(\frac{\partial a}{\partial x}\right)^{2} u^{2} d x+\varepsilon_{4} \int_{\alpha}^{\beta}\left(\mathfrak{J}_{x}(b u)\right)^{2} d x\right] .
\end{aligned}
$$

Integrating (3.5) [(3.6)] with respect to $t$ from 0 to $\tau, \tau \in[0, T]$, using inequality (2.13) and applying Assumption 2.1, yields

$$
\begin{gathered}
2 c_{0} \int_{0}^{\tau}\|u(\cdot, t)\|_{L^{2}(\alpha, \beta)}^{2} d t+\|u(\cdot, \tau)\|_{B_{2}^{1}(\alpha, \beta)}^{2} \\
\leq \int_{0}^{T}\|f(\cdot, t)\|_{B_{2}^{1}(\alpha, \beta)}^{2} d t+\|\varphi\|_{B_{2}^{1}(\alpha, \beta)}^{2} \\
\quad+\left(1+\frac{1}{\varepsilon_{2}}+\varepsilon_{1} c_{3}^{2}\right) \int_{0}^{\tau}\|u(\cdot, t)\|_{B_{2}^{1}(\alpha, \beta)}^{2} d t \\
\quad+\left(\frac{1}{\varepsilon_{1}}+\frac{\varepsilon_{2} c_{4}^{2}(\beta-\alpha)^{2}}{2}\right) \int_{0}^{\tau}\|u(\cdot, t)\|_{L^{2}(\alpha, \beta)}^{2} d t, \\
{\left[c_{0}\|u(\cdot, \tau)\|_{L^{2}(\alpha, \beta)}^{2}+\left\|\frac{\partial u(\cdot, \tau)}{\partial t}\right\|_{B_{2}^{1}(\alpha, \beta)}^{2}\right.} \\
\leq \int_{0}^{\tau}\|f(\cdot, t)\|_{B_{2}^{1}(\alpha, \beta)}^{2} d t+c_{1}\|\varphi\|_{L^{2}(\alpha, \beta)}^{2}+\|\mathcal{x}\|_{B_{2}^{1}(\alpha, \beta)}^{2}
\end{gathered}
$$




$$
\begin{aligned}
& +\left(c_{2}+\varepsilon_{3} c_{3}^{2}+\frac{\varepsilon_{4} c_{4}^{2}(\beta-\alpha)^{2}}{2}\right) \int_{0}^{\tau}\|u(\cdot, t)\|_{L^{2}(\alpha, \beta)}^{2} d t \\
& \left.+\left(1+\frac{1}{\varepsilon_{3}}+\frac{1}{\varepsilon_{4}}\right) \int_{0}^{T}\left\|\frac{\partial u(\cdot, t)}{\partial t}\right\|_{B_{2}^{1}(\alpha, \beta)}^{2} d t\right] .
\end{aligned}
$$

It follows by choosing $\varepsilon_{1}=2 / c_{0}, \varepsilon_{2}=c_{0} / c_{4}^{2}(\beta-\alpha)^{2}, \varepsilon_{3}=\varepsilon_{4}=1$, that

$$
\begin{aligned}
& \int_{0}^{\tau}\|u(\cdot, t)\|_{L^{2}(\alpha, \beta)}^{2} d t+\|u(\cdot, \tau)\|_{B_{2}^{1}(\alpha, \beta)}^{2} \\
& \leq c_{5}\left(\int_{0}^{\tau}\|f(\cdot, t)\|_{B_{2}^{1}(\alpha, \beta)}^{2} d t+\|\varphi\|_{B_{2}^{1}(\alpha, \beta)}^{2}\right)+c_{6} \int_{0}^{\tau}\|u(\cdot, t)\|_{B_{2}^{1}(\alpha, \beta)}^{2} d t \\
& {\left[\|u(\cdot, \tau)\|_{L^{2}(\alpha, \beta)}^{2}+\left\|\frac{\partial u(\cdot, \tau)}{\partial t}\right\|_{B_{2}^{1}(\alpha, \beta)}^{2}\right.} \\
& \leq c_{5}^{\prime}\left(\int_{0}^{\tau}\|f(\cdot, t)\|_{B_{2}^{1}(\alpha, \beta)}^{2} d t+\|\varphi\|_{L^{2}(\alpha, \beta)}^{2}+\|\mathcal{X}\|_{B_{2}^{1}(\alpha, \beta)}^{2}\right) \\
& \left.\quad+c_{6}^{\prime} \int_{0}^{\tau}\left(\|u(\cdot, t)\|_{L^{2}(\alpha, \beta)}^{2}+\left\|\frac{\partial u(\cdot, t)}{\partial t}\right\|_{B_{2}^{1}(\alpha, \beta)}^{2}\right) d t\right]
\end{aligned}
$$

where

$$
\begin{gathered}
c_{5}=\frac{1}{\min \left(c_{0}, 1\right)}\left[c_{5}^{\prime}=\frac{\max \left(1, c_{1}\right)}{\min \left(c_{0}, 1\right)}\right], \\
c_{6}=\frac{1+c_{4}^{2}(\beta-\alpha)^{2} / c_{0}+2 c_{3}^{2} / c_{0}}{\min \left(c_{0}, 1\right)}\left[c_{6}^{\prime}=\frac{\max \left(2, c_{2}+c_{3}^{2}+c_{4}^{2}(\beta-\alpha)^{2} / 2\right)}{\min \left(c_{0}, 1\right)}\right] .
\end{gathered}
$$

Gronwall's lemma implies that

$$
\begin{aligned}
& \int_{0}^{\tau}\|u(\cdot, t)\|_{L^{2}(\alpha, \beta)}^{2} d t+\|u(\cdot, \tau)\|_{B_{2}^{1}(\alpha, \beta)}^{2} \\
& \quad \leq c_{5} \exp \left(c_{6} T\right)\left(\int_{0}^{T}\|f(\cdot, t)\|_{B_{2}^{1}(\alpha, \beta)}^{2} d t+\|\varphi\|_{B_{2}^{1}(\alpha, \beta)}^{2}\right), \\
& \quad\left[\|u(\cdot, \tau)\|_{L^{2}(\alpha, \beta)}^{2}+\left\|\frac{\partial u(\cdot, \tau)}{\partial t}\right\|_{B_{2}^{1}(\alpha, \beta)}^{2}\right. \\
& \left.\quad \leq c_{5}^{\prime} \exp \left(c_{6}^{\prime} T\right)\left(\int_{0}^{T}\|f(\cdot, t)\|_{B_{2}^{1}(\alpha, \beta)}^{2} d t+\|\varphi\|_{L^{2}(\alpha, \beta)}^{2}+\|\mathcal{L}\|_{B_{2}^{1}(\alpha, \beta)}^{2}\right)\right] .
\end{aligned}
$$

Since the right-hand side of (3.10) [(3.11)] is independent of $\tau$, we take the supremum with respect to $T$ from 0 to $T$ in the left-hand side, thus obtaining (2.18) [(2.19)], with $c=c_{5}^{1 / 2} \exp \left(c_{6} T / 2\right)\left[c^{\prime}=c_{5}^{\prime 1 / 2} \exp \left(c_{6}^{\prime} T / 2\right)\right]$. 
REMARK 3.1. We note that if we take the scalar product in the classical space $L^{2}\left(0, T ; L^{2}(\alpha, \beta)\right)$ to obtain a priori estimate, like (2.18) [(2.19)], for a solution of problem (2.4), (2.5), and (2.6) [(2.7), (2.8), and (2.9)] then we are led to construct an integrodifferential operator of multiplication, such that $M u=(\beta-x)(\partial u / \partial t)+\mathfrak{J}_{x}(\partial u / \partial t)$, or $M u=(x-\alpha)\left(\mathfrak{I}_{x}^{*}(\partial u / \partial t)+a(\partial u / \partial x)\right)\left[M u=\mathfrak{I}_{x}((x-\xi)(\partial u(\xi, t) / \partial t))\right]$, where $\mathfrak{I}_{x}^{*}$ is the adjoint of $\mathfrak{J}_{x}$. For details, see $[1,2,4]$.

Proposition 3.2. The operator $L\left[L^{\prime}\right]$ from $B\left[B^{\prime}\right]$ into $F\left[F^{\prime}\right]$ has a closure.

The proof of the proposition is analogous to that of [3, Proposition 1]. Theorem 2.6 can be extended to cover generalized solutions by taking a limit.

COROLLARY 3.3. Under the conditions of Theorem 2.6, there is a constant $c>0$ independent of $u$ such that

$$
\begin{gathered}
\|u\|_{B} \leq c\|\bar{L} u\|_{F}, \quad \forall u \in D(\bar{L}), \\
{\left[\|u\|_{B^{\prime}} \leq c^{\prime}\left\|\bar{L}^{\prime} u\right\|_{F^{\prime}}, \quad \forall u \in D\left(\bar{L}^{\prime}\right)\right] .}
\end{gathered}
$$

This corollary implies the following.

COROLlary 3.4. A generalized solution of (2.4), (2.5), and (2.6) [(2.7), (2.8), and (2.9)] is unique, if it exists, and depends continuously on $(f, \varphi)[(f, \varphi, \varkappa)]$ if $(f, \varphi)[(f, \varphi, \varkappa)]$ is considered in the topology of $F\left[F^{\prime}\right]$ and the solution $u$ is considered in the topology of $B\left[B^{\prime}\right]$.

COROLLARY 3.5. The range $R(\bar{L})\left[R\left(\bar{L}^{\prime}\right)\right]$ is closed in $F\left[F^{\prime}\right]$ and $\overline{R(L)}=R(\bar{L})\left[\overline{R\left(L^{\prime}\right)}=\right.$ $\left.R\left(\bar{L}^{\prime}\right)\right]$.

Hence, to prove the existence of a generalized solution of problem (2.4), (2.5), and (2.6) [(2.7), (2.8), and (2.9)] for all $(f, \varphi) \in F\left[(f, \varphi, \mathcal{\varkappa}) \in F^{\prime}\right]$, it remains to prove that $R(L)\left[R\left(L^{\prime}\right)\right]$ is dense in $F\left[F^{\prime}\right]$.

4. Existence of the solution. In this section, we prove Theorem 2.7, which guarantees that problem (2.4), (2.5), and (2.6) [(2.7), (2.8), and (2.9)] admits a generalized solution.

Proof OF TheOrem 2.7. Let $D_{0}(L)\left[D_{0}\left(L^{\prime}\right)\right]$ be the set of all $u \in D(L)\left[D\left(L^{\prime}\right)\right]$ such that $\ell u=0\left[\ell_{1} u=\ell_{2} u=0\right]$ and let $\mathscr{L}_{0}\left[\mathscr{L}_{0}^{\prime}\right]$ be the principal part of $\mathscr{L}\left[\mathscr{L}^{\prime}\right]$, that is,

$$
\begin{gathered}
\mathscr{L}_{0} u=\frac{\partial u}{\partial t}-\frac{\partial}{\partial x}\left(a(x, t) \frac{\partial u}{\partial x}\right), \\
{\left[\mathscr{L}_{0}^{\prime} u=\frac{\partial^{2} u}{\partial t^{2}}-\frac{\partial}{\partial x}\left(a(x, t) \frac{\partial u}{\partial x}\right)\right] .}
\end{gathered}
$$

We first prove this for which $L\left[L^{\prime}\right]$ is $L_{0}=\left(\mathscr{L}_{0}, \ell\right)\left[L_{0}^{\prime}=\left(\mathscr{L}_{0}^{\prime}, \ell_{1}, \ell_{2}\right)\right]$ with domain $D_{0}(L)=D_{0}\left(L_{0}\right)\left[D_{0}\left(L^{\prime}\right)=D_{0}\left(L_{0}^{\prime}\right)\right]$. Thus, we establish the following lemma. 
LEMMA 4.1. If $\omega\left[\omega^{\prime}\right] \in L^{2}\left(0, T ; B_{2}^{1}(\alpha, \beta)\right)$ is orthogonal to $R\left(L_{0}\right)\left[R\left(L_{0}^{\prime}\right)\right]$ such that

$$
\begin{gathered}
\left(\mathscr{L}_{0} u, \omega\right)_{L^{2}\left(0, T ; B_{2}^{1}(\alpha, \beta)\right)}=0, \quad \forall u \in D_{0}\left(L_{0}\right), \\
{\left[\left(\mathscr{L}_{0}^{\prime} u, \omega^{\prime}\right)_{L^{2}\left(0, T ; B_{2}^{1}(\alpha, \beta)\right)}=0, \forall u \in D_{0}\left(L_{0}^{\prime}\right)\right],}
\end{gathered}
$$

then $\omega\left[\omega^{\prime}\right]$ vanishes almost everywhere in $(\alpha, \beta) \times(0, T)$.

Proof OF Lemma 4.1. Equality (4.2) [(4.3)] can be written as follows:

$$
\begin{gathered}
\left(\frac{\partial u}{\partial t}, \omega\right)_{L^{2}\left(0, T ; B_{2}^{1}(\alpha, \beta)\right)}=\left(\frac{\partial}{\partial x}\left(a \frac{\partial u}{\partial x}\right), \omega\right)_{L^{2}\left(0, T ; B_{2}^{1}(\alpha, \beta)\right)}, \\
{\left[\left(\frac{\partial^{2} u}{\partial t^{2}}, \omega^{\prime}\right)_{L^{2}\left(0, T ; B_{2}^{1}(\alpha, \beta)\right)}=\left(\frac{\partial}{\partial x}\left(a \frac{\partial u}{\partial x}\right), \omega^{\prime}\right)_{L^{2}\left(0, T ; B_{2}^{1}(\alpha, \beta)\right)}\right] .}
\end{gathered}
$$

In (4.4) [(4.5)], we put

$$
\begin{gathered}
u=\mathfrak{J}_{t}\left(e^{c \tau} z\right)=\int_{0}^{t} e^{c \tau} z(x, \tau) d \tau, \\
{\left[u=\mathfrak{J}_{t}^{2} z^{\prime}=\int_{0}^{t}(t-\tau) z^{\prime}(x, \tau) d \tau\right],}
\end{gathered}
$$

where $c$ is a constant such that

$$
c c_{0}-c_{2} \geq 0
$$

$z, \mathfrak{J}_{t}\left(e^{c \tau} z\right), a\left(\partial \mathfrak{I}_{t}\left(e^{c \tau} z\right) / \partial x\right) \in L^{2}\left(0, T ; L^{2}(\alpha, \beta)\right)$, and $z$ satisfies conditions (2.9) $\left[\mathfrak{J}_{t}^{p} z^{\prime}(p=0,1,2), a\left(\partial \mathfrak{I}_{t}^{2} z^{\prime} / \partial x\right) \in L^{2}\left(0, T ; L^{2}(\alpha, \beta)\right)\right.$, and $z^{\prime}$ satisfies conditions (2.17)]. Substituting (4.6) [(4.7)] in (4.4) [(4.5)], yields

$$
\begin{gathered}
\left(e^{c \tau} z, \omega\right)_{L^{2}\left(0, T ; B_{2}^{1}(\alpha, \beta)\right)}=\left(\frac{\partial}{\partial x}\left(a \frac{\partial \mathfrak{J}_{t}\left(e^{c \tau} z\right)}{\partial x}\right), \omega\right)_{L^{2}\left(0, T ; B_{2}^{1}(\alpha, \beta)\right)}, \\
{\left[\left(z^{\prime}, \omega^{\prime}\right)_{L^{2}\left(0, T ; B_{2}^{1}(\alpha, \beta)\right)}=\left(\frac{\partial}{\partial x}\left(a \frac{\partial \mathfrak{J}_{t}^{2} z^{\prime}}{\partial x}\right), \omega^{\prime}\right)_{L^{2}\left(0, T ; B_{2}^{1}(\alpha, \beta)\right)}\right] .}
\end{gathered}
$$

The left-hand side of (4.9) [(4.10)] shows that the mapping

$$
\begin{aligned}
& L^{2}\left(0, T ; B_{2}^{1}(\alpha, \beta)\right) \ni z \rightarrow\left(\frac{\partial}{\partial x}\left(a \frac{\partial \mathfrak{I}_{t}\left(e^{c \tau} z\right)}{\partial x}\right), \omega\right)_{L^{2}\left(0, T ; B_{2}^{1}(\alpha, \beta)\right)}, \\
& {\left[L^{2}\left(0, T ; B_{2}^{1}(\alpha, \beta)\right) \ni z^{\prime} \rightarrow\left(\frac{\partial}{\partial x}\left(a \frac{\partial \mathfrak{I}_{t}^{2} z^{\prime}}{\partial x}\right), \omega^{\prime}\right)_{L^{2}\left(0, T ; B_{2}^{1}(\alpha, \beta)\right)}\right]}
\end{aligned}
$$


is continuous linear function of $z$. From the right-hand side of equality (4.9) [(4.10)], this is true if the function $\omega\left[\omega^{\prime}\right]$ has the following properties:

$$
\begin{gathered}
\mathfrak{I}_{t}^{*}\left(a \mathfrak{I}_{x} \omega\right), \frac{\partial \mathfrak{I}_{t}^{*}\left(a \mathfrak{I}_{x} \omega\right)}{\partial x} \in L^{2}\left(0, T ; L^{2}(\alpha, \beta)\right), \quad \mathfrak{I}_{\beta} \omega=0, \\
{\left[\mathfrak{I}_{t}^{*^{2}}\left(a \mathfrak{I}_{x} \omega^{\prime}\right), \frac{\partial \mathfrak{I}_{t}^{*^{2}}\left(a \mathfrak{I}_{x} \omega^{\prime}\right)}{\partial x} \in L^{2}\left(0, T ; L^{2}(\alpha, \beta)\right), \mathfrak{I}_{\beta} \omega^{\prime}=0\right] .}
\end{gathered}
$$

For given $\omega\left[\omega^{\prime}\right]$ we introduce the function

$$
\begin{gathered}
y(x, t)=-\mathfrak{I}_{x}\left(\frac{\mathfrak{J}_{\xi} \omega}{\beta-\xi}\right), \\
{\left[y^{\prime}(x, t)=-e^{c t}\left(c \mathfrak{I}_{x}^{2} \omega^{\prime}+\frac{\partial \mathfrak{I}_{x}^{2} \omega^{\prime}}{\partial t}\right), \omega^{\prime}(x, 0)=0\right],}
\end{gathered}
$$

here $c$ is a constant verifying condition (4.8). Differentiating (4.13) [(4.14)] with respect to $x$, we obtain

$$
\begin{gathered}
\frac{\partial y}{\partial x}=-\frac{\mathfrak{J}_{x} \omega}{\beta-x}, \quad \frac{\partial^{2} z}{\partial x^{2}}=-\frac{\mathfrak{I}_{x} \omega}{(\beta-x)^{2}}-\frac{\omega}{\beta-x} \\
{\left[\frac{\partial y^{\prime}}{\partial x}=-e^{c t}\left(c \mathfrak{J}_{x} \omega^{\prime}+\frac{\partial \mathfrak{J}_{x} \omega^{\prime}}{\partial t}\right), \quad \frac{\partial^{2} z^{\prime}}{\partial x^{2}}=-e^{c t}\left(c \omega^{\prime}+\frac{\partial \omega^{\prime}}{\partial t}\right)\right]}
\end{gathered}
$$

which implies that

$$
\begin{gathered}
\omega=-\frac{\partial}{\partial x}\left((\beta-x) \frac{\partial y}{\partial x}\right), \\
{\left[\omega^{\prime}=-e^{-c t} \frac{\partial^{2} \mathfrak{I}_{t} y^{\prime}}{\partial x^{2}}\right],} \\
\frac{\partial y(\alpha, t)}{\partial x}=0, \\
{\left[\frac{\partial y^{\prime}(\alpha, t)}{\partial x}=0\right] .}
\end{gathered}
$$

We now replace $\omega\left[\omega^{\prime}\right]$ in (4.9) [(4.10)] by its representation (4.16) [(4.17)], this yields

$$
\begin{aligned}
& -\left(e^{c t} z, \frac{\partial}{\partial x}\left((\beta-x) \frac{\partial y}{\partial x}\right)\right)_{L^{2}\left(0, T ; B_{2}^{1}(\alpha, \beta)\right)} \\
& =-\left(\frac{\partial}{\partial x}\left(a \frac{\partial \mathfrak{I}_{t}\left(e^{c \tau} z\right)}{\partial x}\right), \frac{\partial}{\partial x}\left((\beta-x) \frac{\partial y}{\partial x}\right)\right)_{L^{2}\left(0, T ; B_{2}^{1}(\alpha, \beta)\right)}, \\
& {\left[-\left(z^{\prime}, e^{-c t} \frac{\partial^{2} \mathfrak{I}_{t} y^{\prime}}{\partial x^{2}}\right)_{L^{2}\left(0, T ; B_{2}^{1}(\alpha, \beta)\right)}\right.} \\
& \left.=-\left(\frac{\partial}{\partial x}\left(a \frac{\partial \mathfrak{I}_{t}^{2} y^{\prime}}{\partial x}\right), e^{-c t} \frac{\partial^{2} \mathfrak{I}_{t} z^{\prime}}{\partial x^{2}}\right)_{L^{2}\left(0, T ; B_{2}^{1}(\alpha, \beta)\right)}\right] .
\end{aligned}
$$


In (4.20) [(4.21)] setting $y=z\left[y^{\prime}=z^{\prime}\right]$, and integrating by parts in the right-hand side of (4.20) [(4.21)] with respect to $x$ and $t$ by taking into account (2.6) [(2.9)] and the fact that $u \in D_{0}(L)\left[D_{0}\left(L^{\prime}\right)\right]$, respectively,

$$
\begin{aligned}
-( & \left.\frac{\partial}{\partial x}\left(a \frac{\partial \mathfrak{I}_{t}\left(e^{c T} z\right)}{\partial x}\right), \frac{\partial}{\partial x}\left((\beta-x) \frac{\partial z}{\partial x}\right)\right)_{L^{2}\left(0, T ; B_{2}^{1}(\alpha, \beta)\right)} \\
= & -\left(a \frac{\partial \mathfrak{I}_{t}\left(e^{c T} z\right)}{\partial x},(\beta-x) \frac{\partial z}{\partial x}\right)_{L^{2}\left(0, T ; B_{2}^{1}(\alpha, \beta)\right)} \\
= & -\frac{1}{2} \int_{\alpha}^{\beta} e^{-c T} a(x, T)(\beta-x)\left(\frac{\partial \mathfrak{J}_{T}\left(e^{c t} z\right)}{\partial x}\right)^{2} d x \\
& -\frac{1}{2} \int_{0}^{T} \int_{\alpha}^{\beta}\left(c a(x, t)-\frac{\partial a}{\partial t}\right)(\beta-x)\left(\frac{\partial \mathfrak{I}_{t}\left(e^{c T} z\right)}{\partial x}\right)^{2} d x d t \\
-( & \left.\frac{\partial}{\partial x}\left(a \frac{\partial \mathfrak{I}_{t}^{2} z^{\prime}}{\partial x}\right), e^{-c t} \frac{\partial^{2} \mathfrak{I}_{t} z^{\prime}}{\partial x^{2}}\right)_{L^{2}\left(0, T ; B_{2}^{1}(\alpha, \beta)\right)} \\
= & -\left(a \frac{\partial \mathfrak{I}_{t}^{2} z^{\prime}}{\partial x}, e^{-c t} \frac{\partial \mathfrak{I}_{t} z^{\prime}}{\partial x}\right)_{L^{2}\left(0, T ; B_{2}^{1}(\alpha, \beta)\right)} \\
= & -\frac{1}{2} \int_{\alpha}^{\beta} e^{-c T} a(x, T)\left(\frac{\partial \mathfrak{I}_{T}^{2} z^{\prime}}{\partial x}\right)^{2} d x \\
& \left.-\frac{1}{2} \int_{0}^{T} \int_{\alpha}^{\beta} e^{-c t}\left(c a(x, t)-\frac{\partial a}{\partial t}\right)\left(\frac{\partial \mathfrak{I}_{t}^{2} z^{\prime}}{\partial x}\right)^{2} d x d t\right] .
\end{aligned}
$$

Under Assumption 2.1, we obtain

$$
\begin{aligned}
& -\left(e^{c t} z, \frac{\partial}{\partial x}\left((\beta-x) \frac{\partial z}{\partial x}\right)\right)_{L^{2}\left(0, T ; B_{2}^{1}(\alpha, \beta)\right)} \\
& \leq-\frac{1}{2}\left(c c_{0}-c_{2}\right) \int_{0}^{T} \int_{\alpha}^{\beta}(\beta-x)\left(\frac{\partial \mathfrak{J}_{t}\left(e^{c t} z\right)}{\partial x}\right)^{2} d x d t \leq 0, \\
& {\left[-\left(z^{\prime}, e^{-c t} \frac{\partial^{2} \mathfrak{J}_{t} z^{\prime}}{\partial x^{2}}\right)_{L^{2}\left(0, T ; B_{2}^{1}(\alpha, \beta)\right)}\right.} \\
& \left.\leq-\frac{1}{2}\left(c c_{0}-c_{2}\right) \int_{0}^{T} \int_{\alpha}^{\beta}\left(\frac{\partial \mathfrak{I}_{t}^{2} z^{\prime}}{\partial x}\right)^{2} d x d t \leq 0\right] .
\end{aligned}
$$

Similarly, integrating, by parts, in the left-hand side of (4.23) [(4.24)] with respect to $x$, we get

$$
\begin{gathered}
\int_{0}^{T} \int_{\alpha}^{\beta} e^{c t}(\beta-x) z^{2} d x d t \leq 0 \\
{\left[\int_{\alpha}^{\beta} e^{-c T}\left(\mathfrak{J}_{T} z^{\prime}\right)^{2} d x+\int_{0}^{T} \int_{\alpha}^{\beta} e^{-c t}\left(\mathfrak{J}_{t} z^{\prime}\right)^{2} d x d t \leq 0\right],}
\end{gathered}
$$


and thus $z \equiv 0\left[z^{\prime} \equiv 0\right]$ almost everywhere in $(\alpha, \beta) \times(0, T)$. Hence $\omega \equiv 0\left[\omega^{\prime} \equiv 0\right]$ almost everywhere in $(\alpha, \beta) \times(0, T)$.

Now, we return to the proof of Theorem 2.7. We have already noted that it is sufficient to prove that the range $R(L)\left[R\left(L^{\prime}\right)\right]$ is dense in $F\left[F^{\prime}\right]$. Let $W=\left(\omega, \omega_{0}\right)\left[W^{\prime}=\right.$ $\left.\left(\omega^{\prime}, \omega_{1}, \omega_{2}\right)\right]$ be orthogonal to $R\left(L_{0}\right)\left[R\left(L_{0}^{\prime}\right)\right]$, so that

$$
\begin{gathered}
\left(\mathscr{L}_{0} u, \omega\right)_{L^{2}\left(0, T ; B_{2}^{1}(\alpha, \beta)\right)}+\left(\ell u, \omega_{0}\right)_{B_{2}^{1}(\alpha, \beta)}=0, \quad \forall u \in D\left(L_{0}\right), \\
{\left[\left(\mathscr{L}_{0}^{\prime} u, \omega^{\prime}\right)_{L^{2}\left(0, T ; B_{2}^{1}(\alpha, \beta)\right)}+\left(\ell_{1} u, \omega_{1}\right)_{L^{2}(\alpha, \beta)}+\left(\ell_{2} u, \omega_{2}\right)_{B_{2}^{1}(\alpha, \beta)}=0, \quad \forall u \in D\left(L_{0}^{\prime}\right)\right] .}
\end{gathered}
$$

Assuming that $u$ is any element of $D_{0}\left(L_{0}\right)\left[D_{0}\left(L_{0}^{\prime}\right)\right]$, we have

$$
\begin{gathered}
\left(\mathscr{L}_{0} u, \omega\right)_{L^{2}\left(0, T ; B_{2}^{1}(\alpha, \beta)\right)}=0, \quad \forall u \in D_{0}\left(L_{0}\right), \\
{\left[\left(\mathscr{L}_{0}^{\prime} u, \omega^{\prime}\right)_{L^{2}\left(0, T ; B_{2}^{1}(\alpha, \beta)\right)}=0, \forall u \in D_{0}\left(L_{0}^{\prime}\right)\right] .}
\end{gathered}
$$

Lemma 4.1 implies that $\omega \equiv 0\left[\omega^{\prime} \equiv 0\right]$ almost everywhere in $(\alpha, \beta) \times(0, T)$. Hence, (4.26) and [(4.27)] implies that

$$
\begin{gathered}
\left(\ell u, \omega_{0}\right)_{B_{2}^{1}(\alpha, \beta)}=0, \quad \forall u \in D\left(L_{0}\right), \\
{\left[\left(\ell_{1} u, \omega_{1}\right)_{L^{2}(\alpha, \beta)}+\left(\ell_{2} u, \omega_{2}\right)_{B_{2}^{1}(\alpha, \beta)}=0, \forall u \in D\left(L_{0}^{\prime}\right)\right] .}
\end{gathered}
$$

Since $R(\ell)$ is everywhere dense in $B_{2}^{1}(\alpha, \beta)\left[\ell_{1} u, \ell_{2} u\right.$ are independent, and $R\left(\ell_{1}\right)$ and $R\left(\ell_{2}\right)$ are everywhere dense in $L^{2}(\alpha, \beta)$ and $B_{2}^{1}(\alpha, \beta)$, resp.], (4.29) [(4.30)] implies that $\omega_{0} \equiv 0\left[\omega_{1} \equiv 0\right.$ and $\left.\omega_{2} \equiv 0\right]$. Hence $R\left(L_{0}\right)\left[R\left(L_{0}^{\prime}\right)\right]$ is dense in $F\left[F^{\prime}\right]$.

Now consider the general case. If we use the fact that $R\left(L_{0}\right)\left[R\left(L_{0}^{\prime}\right)\right]$ is dense in $F\left[F^{\prime}\right]$ and $L-L_{0}=\left(\mathscr{L}-\mathscr{L}_{0}, \ell\right)\left[L^{\prime}-L_{0}^{\prime}=\left(\mathscr{L}^{\prime}-\mathscr{L}_{0}^{\prime}, \ell_{1}, \ell_{2}\right)\right]$ maps continuously $B\left[B^{\prime}\right]$ into $F\left[F^{\prime}\right]$, we conclude that we can prove that $R(L)\left[R\left(L^{\prime}\right)\right]$ is dense in $F\left[F^{\prime}\right]$ by means of the method of continuation along parameter. We will not describe the application of this method because it is analogous to the method used, for instance, in [4].

ACKNOWLEDGMENT. This work was supported by the Larbi Ben M'hidi University Centre-Oum El Bouagui, Algeria.

\section{REFERENCES}

[1] A. Bouziani, Solution forte d'un problème mixte avec condition intégrale pour une classe d'équations paraboliques, Maghreb Math. Rev. 6 (1997), 1-17 (French).

[2] __ Solution forte d'un problème mixte avec une condition non locale pour une classe d'équations hyperboliques, Acad. Roy. Belg. Bull. Cl. Sci. (6) 8 (1997), 53-70 (French).

[3] _ Strong solution for a mixed problem with nonlocal condition for certain pluriparabolic equations, Hiroshima Math. J. 27 (1997), 373-390.

[4] __ On a class of parabolic equations with a nonlocal boundary condition, Acad. Roy. Belg. Bull. Cl. Sci. (6) 10 (1999), 61-77.

[5] J. R. Cannon, S. P. Esteva, and J. van der Hoek, A Galerkin procedure for the diffusion equation subject to the specification of mass, SIAM J. Numer. Anal. 24 (1987), 499515. 
[6] J. R. Cannon and J. van der Hoek, An implicit finite difference scheme for the diffusion equation subject to the specification of mass in a portion of the domain, Numerical Solutions of Partial Differential Equations (Parkville, 1981) (J. Noye, ed.), NorthHolland, Amsterdam, 1982, pp. 527-539.

[7] G. Fairweather and R. D. Saylor, The reformulation and numerical solution of certain nonclassical initial-boundary value problems, SIAM J. Sci. Statist. Comput. 12 (1991), no. $1,127-144$.

[8] H. B. Keller, A new difference scheme for parabolic problems, Numerical Solution of Partial Differential Equations, II (SYNSPADE 1970) (Proc. Sympos., Univ. of Maryland, College Park, Md., 1970) (B. Hubbard, ed.), Academic Press, New York, 1971, pp. 327350.

[9] R. W. Lardner, Stability of the numerical solution of a parabolic system with integral subsidiary conditions, Comput. Math. Appl. 19 (1990), no. 12, 41-46.

[10] P. Shi, Weak solution to an evolution problem with a nonlocal constraint, SIAM J. Math. Anal. 24 (1993), 46-58.

AbDelfatah BouZiani: Département de Mathématiques, Centre UniVersitaire LaRbi BEN M'Hidi-OUm El BOUAGUi, B.P. 565, 04000, AlgERIA

Current address: MATHEMATiCAl Division, The Abdus SALAm INTERNATIONAL CENTRE FOR Theoretical Physics (ICTP), Strada Costiera 11, 34100 Trieste, ITALY

E-mail address: bouziani@ictp.trieste.it 


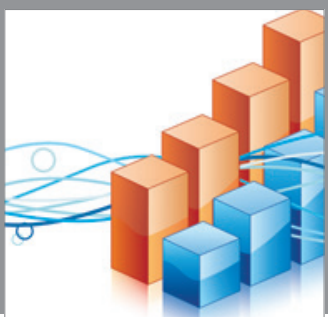

Advances in

Operations Research

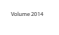

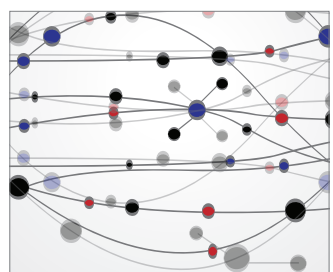

\section{The Scientific} World Journal
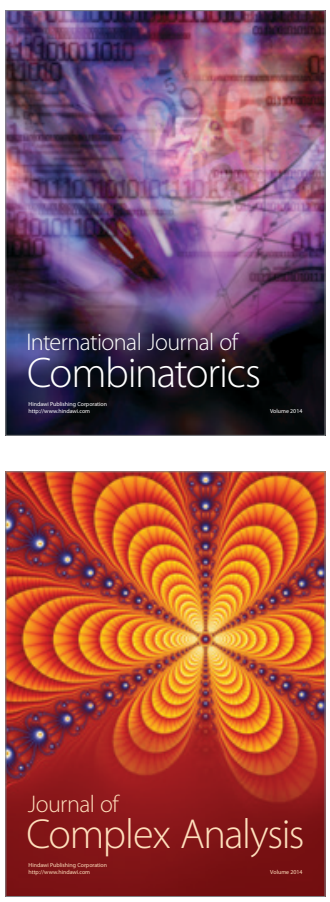

International Journal of

Mathematics and

Mathematical

Sciences
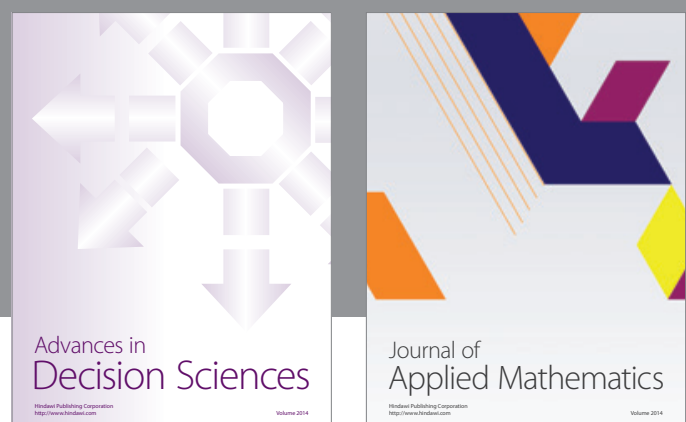

Journal of

Applied Mathematics
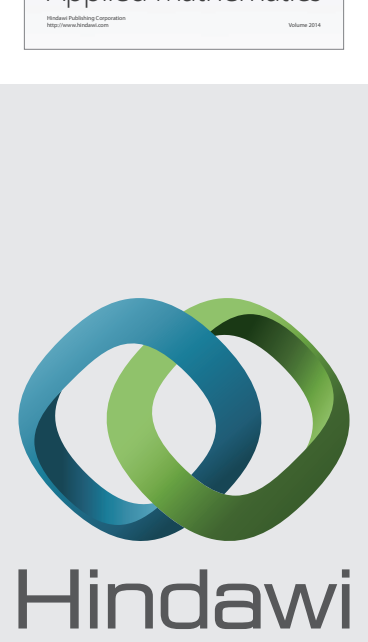

Submit your manuscripts at http://www.hindawi.com
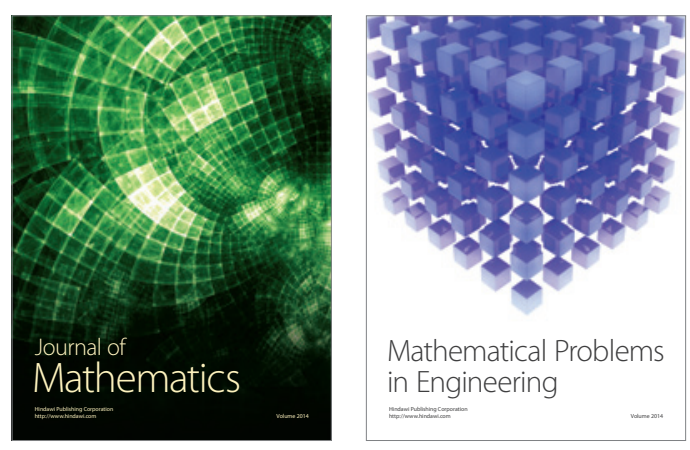

Mathematical Problems in Engineering
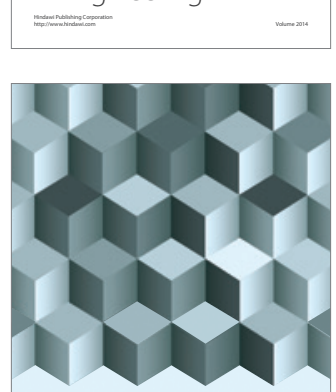

Journal of

Function Spaces
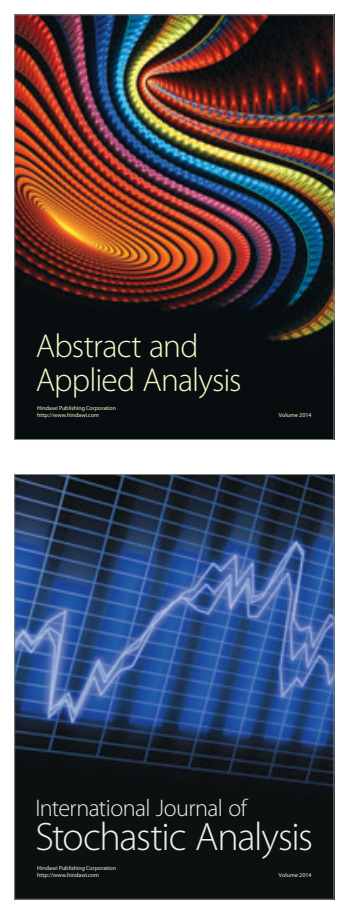

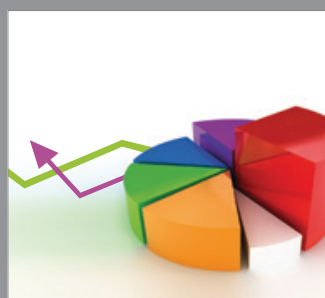

ournal of

Probability and Statistics

Promensencen
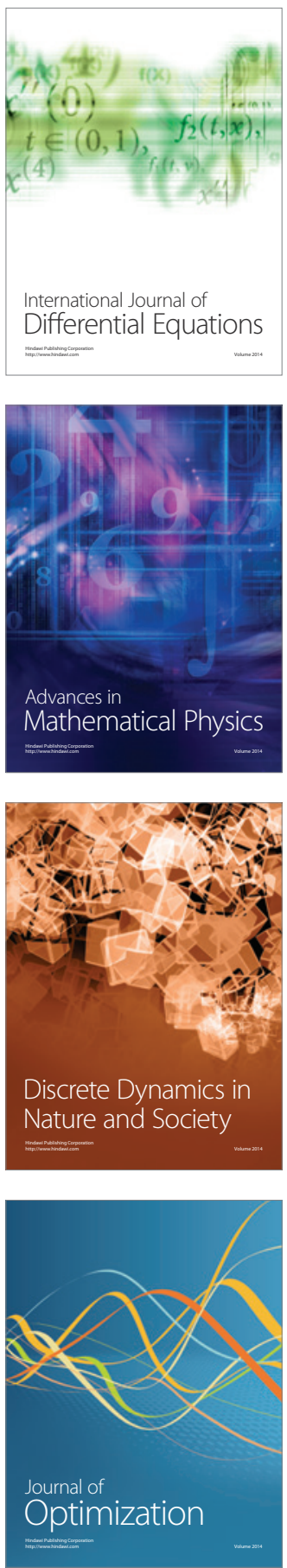